\title{
Dynamics of a two-species model with transitions in population interactions
}

\author{
Yaping Wang* and Jingyu Xiao \\ School of Science, Tianjin University of Technology and Education Tianjin 300222, P. R. China
}

\begin{abstract}
A two-species model with variable population interactions and harvesting on one of the species is studied. Existence and stability of equilibria and existence of periodic solutions are established, existence of some bifurcation phenomena are analytically and numerically studied, explicit threshold values are computed to determine the kind of interaction ( mutualism, competition, host-parasite) between the two species. A brief discussion on the influence of the harvesting function on the dynamics of the model is also included.
\end{abstract}

\section{Introduction}

The population models with static interactions, like competition, predator-prey, mutualism, is widely and well studied in [2], [6], [7], [11], [14]. However, there are some evidences [5], [12], [13], [14] show that it not adequate the case as the interspecific relationship may change depending on population densities and environmental parameters and so on. Several studies [5], [4], [15] have focus on condition interaction which is represented via nonconstant functions. Jorge Rebaza [10] studied the model incorporates linear $\alpha$-functions representing the variable interaction between the species, and also considered harvesting on one of species by an external agent. For the first time, Hopf bifurcations and periodic solutions are found in this kind of models. The aim of this paper is to consider more general and rational $\alpha$-functions representing the variable interaction between the species, and discuss the main influence of harvesting on the dynamics of the model.

In the present paper, we consider the following model:

$$
\left\{\begin{array}{l}
\frac{d u}{d t}=u\left(r_{1}-k_{1} u\right)+\alpha_{1}(u, v) u v-\frac{h u}{e+u} \\
\frac{d v}{d t}=v\left(r_{2}-k_{2} v\right)+\alpha_{2}(u, v) u v
\end{array}\right.
$$

Where $u(t) \geq 0, u(t) \geq 0$ are the population densities of species 1 and 2 respectively, and all the parameters are positive. The constants $r_{1}, r_{2}$ are the corresponding intrinsic growth rates, $k_{1}$ and $k_{2}$ are the coefficients of intraspecific competition. The function $H(u)=\frac{h u}{e+u}$ is the harvesting function which is increasing smoothly with the size $u$ of the population, $h$ is the rate-of-harvesting limit, and $e$ is the number of species 1 it take to reach one half of the maximum harvesting rate. We consider the functions $\alpha_{1}, \alpha_{2}$ represent the interactions between both species, where

$$
\alpha_{1}(u, v)=\frac{a_{1}-b_{1} v}{c_{1}+d_{1} v}, \alpha_{2}(u, v)=\frac{a_{2}-b_{2} u}{c_{2}+d_{2} u}
$$

The parameters $a_{i}, b_{i}, c_{i}, d_{i}(i=1,2)$ representing changes in environmental conditions. So $\alpha_{1}, \alpha_{2}$ can take positive or negative values, and thus the interspecific interactions are not fixed but vary with the environmental parameters and the system state, and can take positive or negative values. Classically, one considers $\alpha_{1}, \alpha_{2}$ as fixed parameters, and their signs determine the interspecific interaction. In particular, if $c_{1}=c_{2}=1$, and $d_{1}=d_{2}=0$, then the model reduces to the one studied in [1], [10].

The paper is organized as follows. In section 2, we studied the existence and local stability of the equilibrium. The existence of certain bifurcations both analytically and numerically is resolved in section 3 . In section 4 , the impact of harvesting on one of the species is discussed.

\section{Local stability of equilibria}

The system (1), (2) determines the following equilibria:

$$
P_{0}=(0,0), P_{1}=\left(u_{ \pm}, 0\right), P_{2}=\left(0, \frac{r_{2}}{k_{2}}\right), P_{3}=\left(u_{3}, v_{3}\right) \text {. }
$$

Here

$$
\begin{aligned}
u_{ \pm} & =\frac{\left(r_{1}-k_{1} e\right) \pm \sqrt{\left(r_{1}-k_{1} e\right)^{2}+4 k_{1}\left(r_{1} e-h\right)}}{2 k_{1}} \\
& =\frac{\left(r_{1}-k_{1} e\right) \pm \sqrt{\left(r_{1}+k_{1} e\right)^{2}-4 k_{1} h}}{2 k_{1}} .
\end{aligned}
$$

Thus $u_{ \pm}$is real if $h \leq \frac{\left(r_{1}+k_{1} e\right)^{2}}{4 k_{1}}$. This means that if the rate-of-harvesting limit above the threshold value in (3), there are no equilibria with species 1 only.

From (3) we can see that: (a) if $\left(r_{1} e-h\right)<0$ and $r_{1}-k_{1} e>0$, then $u_{ \pm}>0$; (b) if $\left(r_{1} e-h\right)>0$, then only

\footnotetext{
* Corresponding author: ypwang1001@163.com
} 
$u_{+}>0$; (c) if $\left(r_{1} e-h\right)<0$ and $r_{1}-k_{1} e<0$, then $u_{ \pm}<0$; So if $h$ is above the value $r_{1} e$, then at the same time the intrinsic growth rate $r_{1}$ of species 1 , has to be above the value $k_{1} e$, to have a well defined equilibrium $\left(u_{ \pm}, 0\right)$.

For $u_{3}>0, v_{3}>0$, the coexistence equilibrium $P_{3}=\left(u_{3}, v_{3}\right)$ is solution of

$$
\left\{\begin{array}{l}
r_{1}-k_{1} u+\frac{a_{1}-b_{1} v}{c_{1}+d_{1} v} v-\frac{h}{e+u}=0 \\
r_{2}-k_{2} v+\frac{a_{2}-b_{2} u}{c_{2}+d_{2} u}=0 .
\end{array}\right.
$$

From the second nullcline in (4), we get $v$ is a convex curve about $u$. So we can establish conditions under which there is at least one solution of (4), with $u_{3}>0$, $v_{3}>0$. For instance, denote by $\mathrm{v} 0$ the v-intercept of the first nullcline in (4), and by $v^{*}$ its minimum value. Let $\frac{r_{2}}{k_{2}}<v^{*}<\frac{a_{1}}{b_{1}}$ and $h \geq r_{1} e$. For this nullcline to intersect at least once with the second nullcline in (4), we impose a negative slope on the first nullcline at the $v$-intercept, that is $h>r_{1} e^{2}$.

The general Jacobian $A_{0}$ of (1):

$$
\left(\begin{array}{cc}
r_{1}-2 k_{1} u+\frac{a_{1}-b_{1} v}{c_{1}+d_{1} v} v-\frac{h}{(e+u)^{2}} & \frac{\left(a_{1} c_{1}-2 b_{1} c_{1} v-b_{1} d_{1} v^{2}\right)}{\left(c_{1}+d_{1} v\right)^{2}} u \\
\frac{\left(a_{2} c_{2}-2 b_{2} c_{2} u-b_{2} d_{2} u^{2}\right)}{\left(c_{2}+d_{2} u\right)^{2}} v & r_{2}-2 k_{2} v+\frac{a_{2}-b_{2} u}{c_{2}+d_{2} u} u
\end{array}\right)
$$

In the theorem below, we denote

$$
h^{*}=r_{1} e\left[1+\frac{k_{2} a_{2}-b_{1} r_{2}}{k_{2}\left(k_{2} c_{1}+d_{1} r_{2}\right)}\right] \text {. }
$$

Theorem 2.1. The boundary equilibrium points of (1) have the following local stability properties:

(a) $P_{0}=(0,0)$ is an unstable node if $h<r_{1} e$ and a saddle if $h>r_{1} e . P_{0}$ is never a focus nor of center type.

(b) $P_{1}=\left(u_{ \pm}, 0\right)$ is a saddle or a stable/unstable node according to

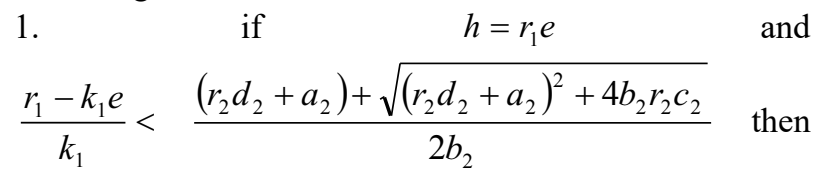
$P_{1}$ is a saddle, and a stable node if the inequality is reversed.

2. if $h<r_{1} e ; r_{1}-k_{1} e>r_{2} d_{2}+a_{2}$ and $\frac{b_{2} r_{2} c_{2}}{r_{1} e-h} \leq k_{1} \leq b_{2}$; then $P_{1}$ is a stable node, and a saddle if all the inequalities but the first one are reversed.

3. if $h>r_{1} e, b_{2}<k_{1}<\frac{1}{2} r_{1}$ and $h+4 k_{1} e<r_{1}-k_{1} e<$ $r_{2} d_{2}+a_{2}$ then $P_{1}$ is a saddle, and an unstable node if $h>r_{1} e, k_{1} \geq \max \left\{b_{2}, \frac{r_{1}}{2}\right\}, r_{1}-k_{1} e<$ $\min \left\{h-r_{1} e, r_{2} d_{2}+a_{2}\right\}$.

4. $P_{1}$ is never a focus nor of center type.

(c) $P_{2}=\left(0, \frac{r_{2}}{k_{2}}\right)$ is a saddle if $h<h^{*}$, and a stable node if $h>h^{*} . P_{2}$ is never a focus nor of center type.

Proof : (a) At $P_{0}$ the eigenvalues are $\lambda_{1}=r_{1}-\frac{h}{e}$ and $\lambda_{2}=r_{2}$, which proves the claim. (b) At $P_{1}$ the eigenvalues of Jacobian are $\lambda_{1}=\frac{e\left(h-r_{1} e\right)+2\left(h-r_{1} e\right) u-r_{1} u^{2}}{(e+u)^{2}}$, $\lambda_{2}=\frac{-b_{2} u^{2}+\left(r_{2} d_{2}+a_{2}\right) u+r_{2} c_{2}}{c_{2}+d_{2} u}$. Therefore, if $h \leq r_{1} e$, $\lambda_{1}<0$.when $h>r_{1} e, \lambda_{1}>0$ if $0<u<u^{\prime}$ and $\lambda_{1}<0$ if $u>u^{\prime}$, where $u^{\prime}=\frac{1}{r_{1}}\left[h-r_{1} e+\sqrt{\left(h-r_{1} e\right)^{2}+e r_{1}\left(h-r_{1} e\right)}\right]$ is the positive root of the parabola. By comparing $u^{\prime}$ with $u=u_{ \pm}$in (3), we see that if $r_{1}<2 k_{1}$ and $r_{1}-k_{1} e<h-r_{1} e$ then $\lambda_{1}>0$,if $r_{1}>2 k_{1}$ and $r_{1}-k_{1} e>h-r_{1} e$ then $\lambda_{1}<0$. In the same way, we get if $h \geq r_{1} e, b_{2}<k_{1}$ and $r_{1}-k_{1} e<\left(r_{2} d_{2}+a_{2}\right)$ then $\lambda_{2}>0$. If $h<r_{1} e, b_{2} \leq k_{1} \leq \frac{b_{2} r_{2} c_{2}}{r_{1} e-h}$ and $r_{1}-k_{1} e<r_{2} d_{2}+a_{2}$, then $\lambda_{2}>0$ and $\lambda_{2}<0$ if all the inequalities are reversed but the first one. Combining the inequalities above, the first three statements follow. In addition, $P_{1}$ is never a focus nor of center type, as none of the eigenvalues is complex.

(c). At $P_{2}$, the eigenvalues of the Jacobian are $\lambda_{1}=r_{1}+\frac{\left(k_{2} a_{2}-b_{1} r_{2}\right) r_{2}}{k_{2}\left(k_{2} c_{1}+d_{1} r_{2}\right)}-\frac{h}{e}$, and $\lambda_{2}=-r_{2}<0$. Thus it is clear that $\lambda_{1}>0$ if $h<h^{*}$ and $\lambda_{1}<0$ if $h>h^{*}$.Clearly, $P_{2}$ is never a focus nor of center type.

\section{Interspecific relationships}

For the coexistence equilibrium $P_{3}=\left(u_{3}, v_{3}\right)$, with $u_{3}>$ $0, v_{3}>0$, the sign the slope of the nullcline (4) at the corresponding equilibrium point indicates the type of the relationship: denoting with $m_{1}, m_{2}$ the slope of $u$ and $v$ nullcline in (4) respectively. We have mutualism if $m_{1}>$ 0 and $m_{2}>0$, competition if $m_{1}<0$ and $m_{2}<0$, hostparasite if $m_{1}>0$ and $m_{2}<0$ and $m_{1}<0$ and $m_{2}>0$. From (4), we get

$$
\begin{gathered}
m_{1}=\frac{\left[k_{1}(e+u)^{2}-h\right]\left(c_{1}+d_{1} v\right)^{2}}{(e+u)^{2}\left(a_{1} c_{1}-2 b_{1} c_{1} v-b_{1} d_{1} v^{2}\right)}, \\
m_{2}=\frac{\left(a_{2} c_{2}-2 b_{2} c_{2} u-b_{2} d_{2} u^{2}\right)}{\left(c_{2}+d_{2} u\right)^{2} k_{2}}
\end{gathered}
$$

For convenience we denote $D_{1}=a_{1} c_{1}-2 b_{1} c_{1} v-b_{1} d_{1} v^{2}$ 
$D_{2}=a_{2} c_{2}-2 b_{2} c_{2} u-b_{2} d_{2} u^{2}, E_{1}=\sqrt{4 b_{1} c_{1}\left(b_{1} c_{1}+a_{1} d_{1}\right)}$,

$E_{2}=\sqrt{4 b_{2} c_{2}\left(b_{2} c_{2}+a_{2} d_{2}\right)}$, and $u^{+}=\frac{E_{1}}{2 b_{1} d_{1}}-\frac{c_{1}}{d_{1}}, v^{+}=$

$\frac{E_{2}}{2 b_{2} d_{2}}-\frac{c_{2}}{d_{2}}$. Then if $e>\sqrt{\frac{h}{k_{1}}}, k_{1}(e+u)^{2}-h>0$, there

is mutualism at $P_{3}$ when $D_{1}>0$ and $D_{2}>0$, that is when $0<v<v^{+}$and $0<u<u^{+}$; there is competition at $P_{3}$ when $u>u^{+}$and $v>v^{+}$and host-parasite at $P_{3}$ when either $u>u^{+}$and $0<v<v^{+}$, or $0<u<u^{+}$and $v>v^{+}$. If instead $e<\sqrt{\frac{h}{k_{1}}}$, then $k_{1}(e+u)^{2}-h>0$ if $u>u_{1}\left(u_{1}=\right.$ $\frac{-k_{1} e+\sqrt{k_{1} h}}{k_{1}}$ and $k_{1}(e+u)^{2}-h<0$ if $0<u<u_{1}$. Then coexistence equilibrium $P_{3}$ represents :

Mutualism when $\quad e>\sqrt{\frac{h}{k_{1}}}, 0<v<v^{+}, 0<u<u^{+}$or $e<\sqrt{\frac{h}{k_{1}}}, v>v^{+}, 0<u<u^{+}$.

Competition when $e>\sqrt{\frac{h}{k_{1}}}, v>v^{+}, u>u^{+} \quad$ or $e<\sqrt{\frac{h}{k_{1}}}, 0<v<v^{+}, u>u^{+}$.

Host-parasite when $e>\sqrt{\frac{h}{k_{1}}}, 0<u<u^{+}, v>v^{+}$ $\left(u>u^{+}, 0<v<v^{+}\right)$or $e<\sqrt{\frac{h}{k_{1}}}, 0<u<u^{+}, 0<v<v^{+}$,

$\left(u>u^{+}, v>v^{+}\right)$.

Remark. These inequalities provide with threshold value that determine the kind of relationship between the species.

In the theorem below, we denote

$$
B=\frac{k_{1}(e+u)^{2}-h}{(e+u)^{2}}
$$

Theorem 2.2 The coexistence equilibrium $P_{3}=\left(u_{3}, v_{3}\right)$ of (1) has the following stability properties:

(a) It is locally asymptotically stable if $B>0$ and $\frac{m_{2}}{m_{1}}<1 \quad$ or $\quad B>0 \quad, \quad B u+k_{2} v>0 \quad, \quad \frac{m_{2}}{m_{1}}>1 \quad$ and $\left(B u+k_{2} v\right)^{2} \leq 4 B k_{2}\left(1-\frac{m_{2}}{m_{1}}\right) u v$.

(b) It is locally asymptotically unstable if $B<0$, $B u+k_{2} v<0, \frac{m_{2}}{m_{1}}>1$ and
$\left(B u+k_{2} v\right)^{2} \leq 4 B k_{2}\left(1-\frac{m_{2}}{m_{1}}\right) u v$.

(c). It is a saddle if $B>0, \frac{m_{2}}{m_{1}}>1$,or $B<0, \frac{m_{2}}{m_{1}}<1$ and $B u+k_{2} v \neq 0$.

(d). It is a focus if $\left(B u+k_{2} v\right)^{2} \leq 4 B k_{2}\left(1-\frac{m_{2}}{m_{1}}\right) u v$. It is stable if $B u+k_{2} v>0$ and unstable if the last inequality is reversed.

(e). It is of center-type if $B u+k_{2} v=0$ and

$$
\left[\left(h<k_{1}(e+u)^{2}\right), \frac{m_{2}}{m_{1}}<1 \text { or }\left(h>k_{1}(e+u)^{2}\right), \frac{m_{2}}{m_{1}}>1\right] \text {. }
$$

Proof. Using the nullcline equations (4), Jacobian at $P_{3}$ can be written as

$$
J\left(u_{3}, v_{3}\right)=\left(\begin{array}{cc}
-B u & \frac{B u}{m_{1}} \\
m_{2} k_{2} v & -k_{2} v
\end{array}\right) .
$$

Thus, the eigenvalues are

$$
\lambda_{1,2}=\frac{-\left(b u+k_{2} v\right) \pm \sqrt{\left(b u+k_{2} v\right)^{2}-4 B k_{2}\left(1-\frac{m_{2}}{m_{1}}\right) u v}}{2} .
$$

Through analysis symbols of $\lambda_{1}$ and $\lambda_{2}$, we confirmed the above conclusions.

\section{Bifurcations}

Equations should be centred and should be numbered with the number on the right-hand side.

Theorem 3.1. The system (1) undergoes a transcritical bifurcation at $P_{0}=(0,0)$ when $h=r_{1} e$ and $r_{1} \neq k_{1} e$, and at $P_{2}=\left(0, \frac{r_{2}}{k_{2}}\right)$ when $h=h^{*}, r_{1}>k_{1} e$ and $k_{2}>k_{2}^{*}=$

$$
\frac{b_{1} c_{1} r_{2}+\sqrt{b_{1}^{2} c_{1}^{2} r_{2}^{2}+a_{1} c_{1} b_{1} d_{1} r_{2}^{2}}}{a_{1} c_{1}} \text {. }
$$

Proof. At $P_{0}$, the Jacobian has one zero eigenvalue if and only if $h=r_{1} e$. When $\lambda_{1}=0$, an eigenvector is $x^{T}$ $=[1 ; 0]$, and a left eigenvector is also $y^{T}=[1 ; 0]$. If we write the right hand side of $(1)$ as $f(u, v)$, then one gets $y^{T} f_{h}\left(P_{0}\right)=0, y^{T} D f_{h}\left(P_{0}\right) x=-\frac{1}{e} \neq 0, y^{T} D^{2} f_{h}\left(P_{0}\right)(x, x)$ $=\frac{2}{e}\left(r_{1}-k_{1} e\right) \neq 0$, Thus, using Sotomayor's theorem one concludes that under the condition above, the system (1) undergoes a transcritical bifurcation at $P_{0}$.

Similarly, at $P_{2}$ :

$\lambda_{1}=r_{1}+\frac{\left(k_{2} a_{2}-b_{1} r_{2}\right) r_{2}}{k_{2}\left(k_{2} c_{1}+d_{1} r_{2}\right)}-\frac{h}{e}$, when $h=h^{*}, \lambda_{1}=0$. 
$\lambda_{2}=-r_{2}<0$. An eigenvector is $x^{T}=\left[1, \frac{a_{2}}{c_{2} k_{2}}\right]$, and a

left eigenvector is also $y^{T}=[1 ; 0]$. So $y^{T} f_{h}\left(P_{2}\right)=0$,

$$
\begin{aligned}
& y^{T} D f_{h}\left(P_{2}\right) x=-\frac{1}{e} \neq 0, \\
& y^{T} D^{2} f_{h}\left(P_{2}\right)(x, x) \\
& =-2 k_{1}+\frac{2 h}{e^{2}}+\frac{2 a_{2}}{c_{2} k_{2}} \frac{k_{2}^{2} a_{1} c_{1}-2 b_{1} c_{1} r_{2} k_{2}-b_{1} d_{1} r_{2}^{2}}{\left(c_{1} k_{2}+d_{1} r_{2}\right)^{2}} .
\end{aligned}
$$

With $h=h^{*}$, we get

$$
y^{T} D^{2} f_{h}\left(P_{2}\right)(x, x)=
$$

$-2 k_{1}+\frac{2 r_{1}}{e}+\frac{2 r_{2}}{e k_{2}} \frac{a_{1} k_{2}-b_{1} r_{2}}{\left(c_{1} k_{2}+d_{1} r_{2}\right)}+\frac{2 a_{2}}{c_{2} k_{2}} \frac{k_{2}^{2} a_{1} c_{1}-2 b_{1} c_{1} r_{2} k_{2}-b_{1} d_{1} r_{2}^{2}}{\left(c_{1} k_{2}+d_{1} r_{2}\right)^{2}}$

Thus, $y^{T} D^{2} f_{h}\left(P_{2}\right)(x, x) \neq 0 \quad$ when $-2 k_{1}+\frac{2 r_{1}}{e}>0$, $a_{1} k_{2}-b_{1} r_{2}>0$, and $k_{2}^{2} a_{1} c_{1}-2 b_{1} c_{1} r_{2} k_{2}-b_{1} d_{1} r_{2}^{2}>0$ which is true when $k_{2}>k_{2}^{*}$ and $r_{1}>k_{1} e$.

Hence, under the given condition, there is a transcritical bifurcation at $P_{2}$.

Theorem 3.2. The system (1) undergoes a saddle-node bifurcation at

1. $P_{1}=(u, 0)$, if $h=k_{1}(e+u)^{2}$ and $r_{2}+\frac{\left(a_{2}-b_{2} u\right) u}{c_{2}+d_{2} u} \neq 0$.

2. $P_{3}=\left(u_{3}, v_{3}\right)$, if any of these cases hold:
(a). $h=k_{1}(e+u)^{2}$, and $\left[\left(m_{2}>0, v>v^{+}\right)\right.$, or $\left(m_{2}<0\right.$, $\left.\left.0<v<v^{+}\right)\right]$.
(b). $h<k_{1}(e+u)^{2}, m_{1}=m_{2}>0$.
(c). $h>k_{1}(e+u)^{2}, m_{1}<0, B u+k_{2} v \neq 0$.

Proof. 1. The general Jacobian at $P_{1}$ reduces to

$$
J=\left(\begin{array}{cc}
-B u & \frac{B u}{m_{1}} \\
0 & r_{2}+\frac{u\left(a_{2}-b_{2} u\right)}{c_{2}+d_{2} u}
\end{array}\right) .
$$

there is a simple zero eigenvalue when $B=0$ and $r_{2}+\frac{u\left(a_{2}-b_{2} u\right)}{c_{2}+d_{2} u} \neq 0 . B=0$ if and only if $h=k_{1}(e+u)^{2}$. For the zero eigenvalue, one can choose right and left eigenvectors as $x^{T}=y^{T}=[1 ; 0]$, and therefore $y^{T} f_{h}\left(P_{1}\right)=-\frac{u}{e+u} \neq 0, y^{T} D^{2} f_{h}\left(P_{1}\right)(x, x)=$ $(1,0)\left(\frac{-2 k_{1} u}{e+u}+u=\frac{-2 k_{1} u}{e+u} \neq 0\right.$.Thus, sotomayor's theorem implies the existence of a saddle-node bifurcation. 2.(a).If $B=0$, the general Jacobian at $P_{3}$ reduces to

$$
J\left(u_{3}, v_{3}\right)=\left(\begin{array}{cc}
0 & 0 \\
m_{2} k_{2} v & -k_{2} v
\end{array}\right)
$$

An eigenvector of the zero eigenvalue is $x^{T}=\left[1, m_{2}\right]$, and a left eigenvector is $y^{T}=[1 ; 0]$. Thus $y^{T} f_{h}\left(P_{3}\right)=$ $-\frac{u}{e+u} \neq 0$ and $y^{T} D^{2} f_{h}\left(P_{3}\right)(x, x)=-\frac{-2 k_{1} u}{e+u}+\frac{2 m_{2} D_{1}}{\left(c_{1}+d_{1} v\right)^{3}}$ $-\frac{2 m_{2}^{2} c_{1} u\left(b_{1} c_{1}+a_{1} d_{1}\right)}{\left(c_{1}+d_{1} v\right)^{3}}$.Then, if $m_{2}>0, v>v^{+}$or $\left(m_{2}<0,0<v<v^{+}\right), y^{T} D^{2} f_{h}\left(P_{3}\right)(x, x) \neq 0$, if $m_{2}>0$ and $0<v<v^{+}$,or $\left(m_{2}<0, v>v^{+}\right)$, we also need $\frac{c_{1}+d_{1} v}{u}<\frac{m_{2} c_{1}\left(b_{1} c_{1}+a_{1} d_{1}\right)}{D_{1}}$.

(b)(c). If $B>0$ and $m_{1}=m_{2}>0$, or $(B<0$ and $\left.m_{1}=m_{2}>0, B u+k_{2} v \neq 0\right)$, an eigenvector of the zero eigenvalue is $x^{T}=\left[1, m_{1}\right]$, a left eigenvector is $y^{T}=\left[1, \frac{B u}{m_{1} k_{2} v}\right]$. Thus $y^{T} f_{h}\left(P_{3}\right)=-\frac{u}{e+u} \neq 0$, $y^{T} D^{2} f_{h}\left(P_{3}\right)(x, x)=-\frac{h u}{(e+u)^{3}}+\frac{2 m_{2}{ }^{2} c_{1} u\left(b_{1} c_{1}+a_{1} d_{1}\right)}{\left(c_{1}+d_{1} v\right)^{3}}-$ $\frac{2 B c_{2} u v\left(b_{2} c_{2}+a_{2} d_{2}\right)}{m_{1} k_{2} v\left(c_{1}+d_{1} v\right)^{3}}$.Then, if $B>0$ and $m_{1}=m_{2}>0$, or $\left(B<0\right.$ and $\left.m_{1}=m_{2}>0, B u+k_{2} v \neq 0\right)$, $y^{T} D^{2} f_{h}\left(P_{3}\right)(x, x) \neq 0$. Sotomayor's theorem implies the existence of a saddle-node bifurcation for (1).

Theorem 3.3. The system (1) undergoes Hopf bifurcateions when $P_{3}$ satisfies conditions to be of center-type, and $v_{3} \neq \frac{a}{2 b}$.

Proof. Though shifting the equilibrium $P_{3}$ to the origin, we obtain the corresponding power series expansions:

$$
\begin{aligned}
& \dot{u}=a_{10} u+a_{01} v+a_{20} u^{2}+a_{11} u v+a_{02} v^{2}+a_{30} u^{3}+a_{12} u v^{2} \\
& +O_{1}\left(|(u, v)|^{4}\right) . \\
& \dot{v}=b_{10} u+b_{01} v+b_{20} u^{2}+b_{11} u v+b_{02} v^{2}+a_{21} u^{2} v+O_{2}\left(|(u, v)|^{4}\right) .
\end{aligned}
$$
where $a_{i j}$ and $b_{i j}$ are the coefficients of the expansions. From the assumptions, we know $D=a_{10}-b_{01}>0$ and $a_{10}+b_{01}=0$. The Lyapunov number is given by

$$
\sigma=\frac{-3 \pi R}{2 a_{01} D^{\frac{3}{2}}}=\frac{-3 b \pi R}{2 u_{3}\left(a-2 b v_{3}\right) D^{\frac{3}{2}}} .
$$

where $\mathrm{R}$ is a long polynomial expression in the terms of the parameters. There are parameter values so that $P_{3}$ satisfies Theorem 2.2(e). For instance, $r_{1}=0.6 ; r_{2}=0.4$; $k_{1}=0.337 ; k_{2}=0.5 ; a_{1}=d_{1}=d_{2}=1 ; b_{1}=1.3 ; a_{2}=$ $0.4 ; b_{2}=1.5 ; \mathrm{c} 1=\mathrm{c} 2=0 ; \mathrm{h}=0.574805 ; \mathrm{e}=0.5$, for which ${ }_{-}>0$, and turn $r_{1}=0.9 ; r_{2}=0.6 ; b_{1}=0.3$ and others keep constant, we have $\sigma<0$. Thus , the system(1) experiments supercritical and subcritical Hopf bifurcations. 


\section{The impact of harvesting.}

Here we discuss the influence of the harvesting function on the dynamics of the model. We compare our model (1) with the classical model with no harvesting.

Boundary equilibria. If the model with no harvesting, the boundary equilibria are $P_{0}=(0,0), P_{1}=\left(\frac{r_{1}}{k_{1}}, 0\right)$, $P_{2}=\left(0, \frac{r_{2}}{k_{2}}\right)$. Comparing with our model, there are not one but up to two equilibria $\left(u_{ \pm}, 0\right)$ on the horizontal axis when $h \leq \frac{\left(r_{1}+k_{1} e\right)^{2}}{4 k_{1}}$. With no harvesting, $P_{0}$ is always an unstable node, and $P_{1}$ is a saddle or a stable node. Whereas with harvesting, $P_{0}$ can also be a saddle, $P_{1}$ can also be unstable node.

Coexistence equilibria. The coexistence equilibria were found to be nodes, saddles or focus. By including harvesting, the coexistence equilibria in our model can be nodes, focus, or even of center type.

Bifurcations. The system with no harvesting undergoes some bifurcations: transcritical, saddle-node bifurcations. Our model has even richer dynamics, not only those bifurcations, but more importantly, Hopf bifurcations.

\section{Acknowledgements.}

The authors gratefully acknowledge the support of the National Natural Science Fundamental of China(NNSFC) though Grant Nos. 11526157, 11526154 , Tianjin Research Program of Application Foundation and Advanced Technology though Grant Nos. 15JCQNJC01600 and Science and Technology Development Foundation of Tianjin University of Technology and Education though Nos. KJ1431. The authors declare that there is no conflict of interest regarding the publication of this paper.

\section{References}

1. B. Zhang, Z.Zhang, Z.Li and Y.Tao, Stability analysis of a twospecies model with transitions between population interactions, J.Theoret.Biol., 248(2007), 145-153.

2. B.Leard and J.Rebaza, Analysis of predator-prey models with continuous threshold harvesting, Appl.Math.Math.Comput, 217(2011), 5265-5278.

3. Z.Li, M.Han and F.D.Chen, Influence of feedback controls on an autonomous Lotka-Volterra competitive system with infinite delays, Nonliear Anal.RWA, 14(2013), 402-413.

4. T.Lara and J.Rebaza, Dynamics of transitions in population interactions, Nonliear Anal.RWA, 13(2012), 1268-1277.
5. M.J.Hernadez, Spatiotemporal dynamics in variable population interaction with density-dependent interaction coefficients, Ecol.Modeling, 214(2008), 3-16.

6. L.Ji and C.Wu, Qualitive analysis of a predatorprey model with constant-rate prey harvesting incorporating a constant prey refuge, Nonl. Anal: Real World Appl., 11(2010), 2285-2295.

7. K.Lan and C.Zhu, Phase portraits of predator-prey systems with harvesting rates, Disc.Cont. Dynam. Syst, 32(2012), 901-933.

8. S.Lefschetz, Stability of Nonlinear Control Systems, Mathematical Biosciences and Engineering, Academic Press, New York, (1965).

9. J.Hale, Ordinary Differential Euqations, J.Wiley and Sons (1980).

10. J.Rebaza, Bifurcations and periodic orbits in variable population interactions, Communication on pure and Applied analysis, 12(2013), 2997-3012.

11. C,Ji and D.Jiang, Persistence and non-persistence of a mutualism system with stochastic perturbation, Disc. Cont.Dynam, 32(2012), 867-889.

12. M.Hernandez and I.Barradas, Variation in the outcome of population interactions: bifurcations and catastrophes, Math. Biol, 46(2003), 671-594.

13. T. Peschak, Currents of Contrast: Life in South Africa's Two Oceans, Struik Publ,(2006).

14. J.H, Cushman and J.F.Addicot, Conditional interzctions in antplant-herbivore mutualism, in:C.R.Huxley,D.F.Cutler, Ant Plant Interactions, Oxford University Press,(1991),92-103.

15. J.Holland and D.DeAngelis, Consumer-resource theory predicts dynamic transitions between outcomes of iinteractions, Ecol.Letters, 12(2009), 1357-1366. 